HISTOIRE

médecine/sciences $1995 ; 11: 1162-4$

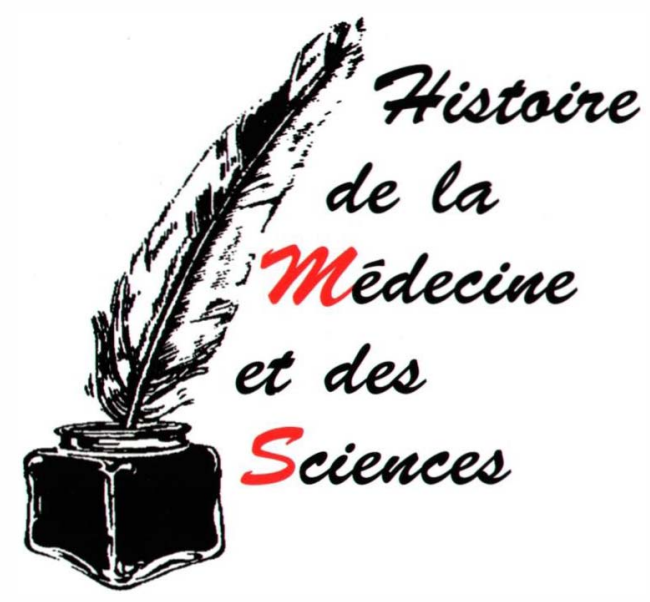

\title{
La première vaccination, deux cents ans, déjà
}

Le 14 mai 1996 a été le deux centième anniversaire de la première vaccination. Edward Jenner, l'auteur de cet exploit, l'un des plus marquants de l'histoire de l'humanité, est né le 17 mai 1749, à Berkeley, non loin de Gloucester, en Angleterre. A cinq ans, déjà orphelin, sans grande fortune, son avenir paraît bouché. Après un essai peu productif de trois années d'études classiques, à 11 ans, il est mis en apprentissage chez un chirurgien, M. J. Ludlow. Il y passe six années, assez pour exercer la chirurgie. A l'époque, aucun diplôme n'était requis. Pourtant, Edward Jenner voulut faire plus. Il partit parfaire ses connaissances d'anatomie et de chirurgie au St-George Hospital de Londres. Il prit pension chez John Hunter et fut certainement très marqué par l'invraisemblable fécondité intellectuelle de ce maître, alliant des compétences d'anatomie, de chirurgie, de dentisterie et surtout d'histoire naturelle. De plus, cette relation lui ouvrit de nombreuses portes, dont celle de Joseph Banks, le chef botaniste de la première expédition de Cook. L'Endeavour, son navire, revenait chargé de gloire et de milliers d'échantillons de flore et de faune, de croquis, de dessins qui devaient être classés. Jenner fut chargé d'une partie de ce travail, qu'il fit à la grande satisfaction de tous. Cook allait repartir et Joseph Banks proposa à Jenner une place de naturaliste, avec de bons appointements, dans le cadre de la nouvelle expédition. Non est auprès de mon frère aîné et des habitants de la vallée de la Severn. L'aventure ne l'attira jamais [1, 2].

Ses études terminées, Jenner réintégra donc sa petite patrie. Cultivé, il pouvait jouer du violon ou de la flûte, versifier et réciter des poèmes et même chanter en public. Cela facilita certainement son intégration dans la société locale. Sa curiosité pour la nature avait été stimulée au cours de son séjour londonien. Il s'adonna à de nombreux travaux d'histoire naturelle. En relation épistolaire suivie avec Hunter, il devint son confident et ami. Il le secondait parfois dans ses recherches, allant sur le terrain recueillir des informations afin de répondre à ses questions. Quelle est la température d'un hérisson en état d'hibernation? Pouvez-vous me procurer des spécimens d'oiseaux migrateurs? Bien sûr, j'aimerais beaucoup recevoir une petite baleine pour ma collection! On ne possède que les lettres de John Hunter, mais elles révèlent les rapports et les intérêts réciproques des deux correspondants. L'élève avait aussi ses idées, ce qui lui vaudra la célèbre réplique: "Pourquoi penser, pourquoi ne pas tenter l'expérience!» «Why to think, why not to trie the experiment ") $[1,3]$.

Jenner avait un esprit curieux et imaginatif. Il fabriqua et lança, quelques mois après le physicien français Charles, un ballon à hydrogène. Il fonda avec quelques amis une société médicale anglaise de province (la première?), entama des travaux de chimie, discerna le premier la calcifi- cation des vaisseaux coronaires à l'autopsie de patients ayant souffert de crises d'angine de poitrine, et bien d'autres choses [1,2]. Sa plus grande réussite fut certainement son étude des mœurs du coucou. Depuis l'Antiquité, on sait que la femelle coucou pond ses œufs, un par un, dans le nid de passereaux, petits oiseaux communs des haies et des buissons. Mais le point le plus étrange de ce parasitisme est la disparition presque immédiate, après l'éclosion du bébé coucou, des autres petits du nid. On croyait que les parents adoptifs jetaient eux-mêmes hors du nid leurs propres enfants pour ne conserver que l'intrus. Jenner observa que c'est le bébé coucou qui, à peine éclos, fait le vide autour de lui en jetant par-dessus bord tous les compétiteurs possibles à l'approvisionnement fourni par les pauvres parents bernés [4]. Cet exploit d'observation, à l'œil nu, valut à Jenner son élection à la Royal Society. Enfin, il se maria avec Catherine Kingscote, demoiselle d'excellente famille, qui renforça sa position dans la société.

Cependant, il vivait de son activité de chirurgien. Point notable, peu de temps avant qu'il ne s'installât à Berkeley, l'inoculation de la petite vérole ou variole, la variolisation dans notre langage actuel, s'était développée. Il s'agissait de la transmission de la variole, en principe bénigne, d'individu à individu dans un but de protection, premier système d'immunisation contre cette maladie très redoutée. Elle avait été introduite en 
Europe, au début du XVII" siècle, par une anglaise, Lady Montagu, qui l'avait vu employée à Constantinople. Les principaux inconvénients de cette vaccination primitive étaient, d'une part, l'induction d'une maladie réelle au patient qui, parfois, pouvait mal évoluer et même lui être fatale, d'autre part, celui-ci restait contagieux durant toute la maladie et pouvait contaminer son entourage $[5,6]$. Elle avait été largement améliorée par le chirurgien-apothicaire anglais, Sutton, et sa méthode devenait une pratique presque courante en Grande-Bretagne. Jenner inocula, dans sa clientèle, une nouvelle couche de population, celle du monde agricole. Il s'aperçut que, dans ce milieu, certaines personnes qu'il variolisait, ne contractaient pas cette maladie, même s'il ne l'avait jamais eue auparavant ( $M y$ attention to this singular disease (the Cow Pox) was first excited by observing, that among those whom in the the country I was frequently called to inoculate, many resisted every effort to give them the Small Pox») [7]. Or une rumeur populaire prétendait qu'une maladie des vaches, le cow pox, pouvait aisément se transmettre à l'homme, et le rendre réfractaire aux atteintes de la variole. Edward Jenner était littéralement obsédé par cette idée et, semble-t-il, en parla tant et si bien à ses amis que certains s'en étaient lassés et même le fuyaient [1].

Jenner étudia pendant des années les bases de cette croyance puis il décida de passer aux actes. Un cas de cow pox ayant surgi dans une ferme de sa clientèle, il choisit un petit garçon de 8 ans, James Phipps, en bonne santé, et qui n'avait jamais contracté la variole. Le 14 mai 1796, il l'inocula avec de la lymphe d'une pustule de vaccine prise sur la main de Sarah Nelmes, probablement la fille du propriétaire de la vache laitière du nom de Blossom (fleur) qui l'avait infectée. Deux petites scarifications sur le bras, un peu de lymphe sur les "plaies" et la première vaccination fut faite. Vers le septième jour, le petit James se plaignit d'une douleur à l'aisselle, au neuvième, il se sentit un peu glacé, perdit son appétit, eut un léger mal de tête et passa une nuit un peu agitée mais, le jour suivant, il était parfaitement remis à la grande satisfaction de Jenner. Médicalement, le passage du cow pox d'un humain à un autre humain, était le seul point critique de l'expérience. Il n'avait jamais été tenté, au moins consciemment. Un mois plus tard, le $1^{\mathrm{er}}$ juillet, Jenner variolisa son petit patient qui, à sa grande satisfaction, présenta seulement la petite réaction que faisaient les variolisés déjà immunisés contre la petite vérole, après avoir déjà contracté une variole, soit naturelle, soit artificielle à la suite d'une variolisation [4, 7]. C'était le succès. Il lui apparut vers le 5 ou le 6 juillet 1796 . Il pouvait inoculer (immuniser) un humain au moyen d'une maladie bénigne pour le protéger contre une, beaucoup plus grave, la variole. L'opération fut appelée la vaccination, la lymphe infectée du virus permettant la transmission de la vaccine, fut le fluide-vaccin ou vaccin et la maladie bénigne, la vaccine [8]. Jenner poursuivit quelque temps ses études et envoya un manuscrit pour publication à la Royal Society. Sir Joseph Banks (son rôle dans la première expédition Cook l'avait fait anoblir), le président de cette société, ne fut pas particulièrement convaincu de son travail et le lui renvoya poliment. Jenner, tenace et croyant en sa méthode, publia à compte d'auteur. Le livre parut le 17 septembre 1798. Il faisait soixante-quinze pages et comportait quatre gravures colorées, pour un prix approximatif de $280 \mathrm{FF}$ 1996. Jenner vint lui-même à Londres, emportant avec lui de la vaccine, enfermée dans le conduit d'une plume d'oiseau, scellée à la cire. Il ne trouva qu'indifférence pour sa nouvelle méthode. Finalement, une vieille connaissance datant de son séjour au St-George Hospital, Henry Cline, se servit d'un peu de vaccin pour... créer une inflammation artificielle à un garçon qui souffrait de la hanche, pensant que cela pourrait peut-être lui faire du bien. Heureusement, un ancien médecin de l'hôpital d'inoculation de Londres assista à l'opération. On variolisa, quelque temps après, le petit patient et cela permit de vérifier qu'il était bel et bien immunisé contre la petite vérole. Cline, chirurgien londonien très connu, diffusa largement cette nouvelle. Elle se propagea merveilleusement vite, entraînant la vaccination avec elle. Le premier pays concerné fut Terre-Neuve, dès la fin de l'année 1798 car Jenner envoya du fluide-vaccin à John Clinch, un de ses amis d'enfance. Clinch cumulait les professions de pasteur et de médecin, il vaccina les membres de sa famille et certains de ses fidèles mais il n'entretint pas la souche qui s'éteignit d'elle-même. La vaccination envahit littéralement l'Europe et la côte Est de l'Amérique du Nord entre 1799 et 1802, atteignit l'Inde et Ceylan en 1802, l'Amérique du Sud, de 1803 à 1804 pour arriver en Chine en 1805 [5, 9-11]. Bel exploit pour une méthode préconisée par un chirurgien de campagne, sans vrai diplôme, et qui, de sa vie durant, ne s'est jamais éloigné, semble-t-il, à plus de deux cents kilomètres de son lieu de naissance!

Pourquoi ce succès? D'une part, la variole était une maladie effroyable qui faisait des ravages difficiles à imaginer à notre époque. En moyenne, un dixième des hommes en mourait, et beaucoup d'autres en restaient infirmes ou défigurés pour le reste de leur vie! D'autre part, la variolisation, la seule méthode de protection connue à cette époque, était une épreuve redoutable. En revanche, la vaccination donnait des symptômes légers et semblait procurer une très bonne immunité. Enfin, elle était surtout une méthode dont la valeur protectrice était aisément démontrée par une variolisation. Des centaines, probablement des milliers de contreépreuves, furent alors tentées et montrèrent le bien-fondé de la vaccination jennerienne.

Quel scientifique (et quelle firme pharmaceutique!) ne rêverait-il pas d'un vaccin (la vaccine) prêt à l'emploi, relativement stable, peu toxique par rapport à la méthode en usage (la variolisation) contre une maladie la plus effrayante et la plus cruelle de l'époque (la variole), et dont l'efficacité pourrait être testée par tout médecin ou chirurgien (par une variolisation), dans le cadre de l'éthique la plus rigoureuse du

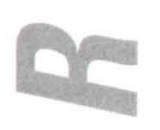

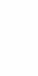

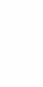

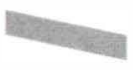


moment, et dans le mois suivant l'immunisation!

Durant les années 1960 et 1970, l'éradication de la variole a été accomplie par des milliers de femmes et d'hommes de bonne volonté, dirigés par l'Organisation Mondiale de la Santé. Le dernier cas recensé l'a été en Somalie, en 1977 [12].

Merci à Edward Jenner qui a enclenché cette bataille victorieuse. Peu de réussites de l'homme peuvent lui être comparées

\section{RÉFÉRENCES}

1. Baron J. The life of Edward Jenner, MD, LLD, FRS, physician extraordinary to his majesty Geo. IV., foreign associate of the National Institute of France, etc. London: Henry Colbrun publisher, 1838, en deux volumes de 624 et 471 pages.

2. Fischer RB. Edward Jenner, 1749-1823. London: André Deutsch, 1991: 361.

3. Harding Rains AJ. Letters from the past, from John Hunter to Edward Jenner. Royal College of Surgeons of England, 1976: 40.
4. Jenner E Observations on the natural history of the Cuckoo, in a letter to J. Hunter, esq, FRS, Philosophical Transactions, 1788, LXXVIII : 219-37.

5. Darmon P. La longue traque de la variole. Paris: Librairie Académique Perrin, $1986: 503$

6. De la Condamine. Histoire de l'inoculation de la petite vérole ou recueil de mémoires, lettres, extraits et autres écrits, sur la petite vérole artificielle, en 2 parties. Amsterdam: La Société Typographique. $1773: 530$.

7. Jenner E. An inquiry into the causes and effects of the variolae vaccinae, a disease discovered in some of the western countries of England, particularly Gloucestershire and known by the name of the cow pox. Printed for the author by Sampson Low, London, 1798: 75

8. Husson HM. Recherches historiques et médicales sur la vaccine. 1801: 130.

9. Comité Central de Vaccine, établi à Paris par la société des souscripteurs pour l'examen de cette découverte, Rapport, à Paris chez Mme Ve Richard, libraire, An XI-1803: 460 .

10. Grassi, Guerin, Archbold, Capelle. Rapport sur la vaccine. Bordeaux: Pinard, An X $(1801): 42$
11. Saunders P Jenner E. The Cheltenham years 1795-1823, being a chronicle of the vaccination campaign. Hanover and London: University Press of New England, 1982: 469

12. Fenner F, Henderson DA, Arita I, Jezek Z, Ladnyi ID. Smallpox and its Eradication. Geneva: World Health Organization, 1988 : 1460 .

\section{Hervé Bazin}

Unité d'Immunologie Expérimentale, Faculté de Médecine, Université de Louvain, Clos Chapelle-aux-Champs 30/56, B-1200 Bruxelles, Belgique.

\section{TIRÉS À PART}

H. Bazin.

\section{BOURSE VOYAGE ÉTUDIANT}

European Cytokine Society

\& Amersham LIFE SCIENCE Biotrak

sont heureux d'annoncer le financement de bourses de voyage étudiant pour le «First Joint meeting of the Intemational Cytokine Society \& the Intemational Society for Interferon and Cytokine Research », qui aura lieu à Genève du 6 au 10 octobre 1996.

Grâce à l'engagement et au soutien d'Amersham Life Science, European Cytokine Society est à même de continuer à promouvoir et encourager l'échange d'informations scientifiques entre les jeunes chercheurs et les experts dans le domaine de la recherche des cytokines.

Les membres de European Cytokine Society, le Conseil et le bureau apprécient l'aide d'Amersham Life Science pour cette première compétition récompensée par un voyage pour ce congrès.

Une dizaine de bourses seront accordées à des étudiants ou candidats en post-doctorat. Les postulants doivent être parrainés par un membre inscrit à European Cytokine Society et soumettre le résumé de leur présentation au Congrès des Cytokines à Genève. Les membres de European Cytokine Society peuvent parrainer deux candidats seulement. Les résumés seront jugés par la Commission scientifique de European Cytokine Society.

\section{Comité des prix}

Jean-Michel DAYER

Alberto MANTOVANI

Didier FRADELIZI

Jacques BERTOGLIO

Michel LEPERS
Président de ECS

Vice-Président de ECS

Secrétaire Trésorier de ECS

Membre de la Commission de ECS

Amersham Life Science 